\title{
Spinal Synaptic Enhancement with Acute Intermittent Hypoxia Improves Respiratory Function after Chronic Cervical Spinal Cord Injury
}

\author{
Francis J. Golder and Gordon S. Mitchell \\ Department of Comparative Biosciences, University of Wisconsin, Madison, Wisconsin 53706
}

\begin{abstract}
Respiratory insufficiency is the leading cause of death after high-cervical spinal cord injuries (SCIs). Although respiratory motor recovery can occur with time after injury, the magnitude of spontaneous recovery is limited. We hypothesized that partial respiratory motor recovery after chronic cervical SCI could be strengthened using a known stimulus for spinal synaptic enhancement, intermittent hypoxia. Phrenic motor output was recorded before and after intermittent hypoxia from anesthetized, vagotomized, and pump-ventilated control and C2 spinally hemisected rats at 2, 4, and 8 weeks after injury. Weak spontaneous phrenic motor recovery was present in all C2-injured rats via crossed spinal synaptic pathways that convey bulbospinal inspiratory premotor drive to phrenic motoneurons on the side of injury. Intermittent hypoxia augmented crossed spinal synaptic pathways [phrenic long-term facilitation; pLTF] for up to 60 min after hypoxia at 8 weeks, but not 2 weeks, after injury. Ketanserin, a serotonin $2 \mathrm{~A}$ receptor antagonist, administered before intermittent hypoxia at 8 weeks after injury prevented pLTF. Serotonergic innervation near phrenic motoneurons was assessed after injury. The limited magnitude of pLTF at 2 weeks was associated with an injury-induced reduction in serotonin-containing nerve terminals in the vicinity of phrenic motoneurons ipsilateral to $\mathrm{C} 2$ hemisection. Thereafter, pLTF magnitude progressively increased with the recovery of serotonergic innervation in the phrenic motor nucleus. Intermittent hypoxia (or pLTF) has intriguing possibilities as a therapeutic tool, because its greatest efficacy may be in patients with chronic SCI, a time when most patients have already achieved maximal spontaneous functional recovery.
\end{abstract}

Key words: hypoxia; phrenic; plasticity; respiration; serotonin; spinal cord injury

\section{Introduction}

Respiratory insufficiency is the leading cause of morbidity and mortality in patients with high-cervical spinal cord injuries (SCIs) (Winslow and Rozovsky, 2003). Thus, strategies to augment respiratory function after SCI may dramatically improve a patient's prognosis, survival, and quality of life. Most attempts to treat SCI focus on preserving spinal pathways during the acute postinjury period (Blight, 2004). In fact, spinal injuries are usually incomplete, with uninjured axons providing a substrate for the weak spontaneous functional recovery often observed in motor systems below the lesion (Raineteau and Schwab, 2001). However, few therapeutic options are available in the chronically injured patient when additional spontaneous functional improvements are unlikely (McDonald et al., 2002).

One well defined model for functional motor recovery below an incomplete high-cervical spinal injury is the crossed phrenic phenomenon (CPP). The CPP is a slow, spontaneous increase in the synaptic strength of existing, but functionally ineffective (i.e.,

Received Jan. 12, 2005; revised Feb. 9, 2005; accepted Feb. 11, 2005.

This work was supported by National Institutes of Health Grant HL 69064 and by the Christopher Reeve Paralysis Foundation (F.J.G.).

Correspondence should be addressed to Dr. Francis J. Golder, Department of Comparative Biosciences, University of Wisconsin, 2015 Linden Drive, Madison, WI 53706. E-mail: golderf@svm.vetmed.wisc.edu. D0I:10.1523/JNEUROSCI.0148-05.2005

Copyright $\odot 2005$ Society for Neuroscience $\quad$ 0270-6474/05/252925-08\$15.00/0 "silent"), synaptic pathways that cross the spinal midline in the midcervical region and innervate contralateral phrenic motoneurons (Goshgarian, 2003). Phrenic motor recovery via crossed spinal pathways enables deeper breaths after injury, although the CPP is slow in onset and is inadequate to completely restore normal breathing (Nantwi et al., 1999; Golder et al., 2001, 2003; Fuller et al., 2003).

Intermittent hypoxia triggers a novel form of spinal serotonin-dependent plasticity, strengthening synaptic pathways to respiratory motoneurons by a mechanism known as respiratory or phrenic long-term facilitation (pLTF) (Mitchell et al., 2001; Baker-Herman and Mitchell, 2002; Baker-Herman et al., 2004). pLTF is expressed as a progressive increase in phrenic motor output despite a return to normal arterial oxygen levels. Spinal serotonin receptor activation is necessary to initiate, but not maintain, pLTF (Fuller et al., 2001a; Baker-Herman and Mitchell, 2002). Serotonin receptor activation, presumably on phrenic motoneurons, initiates new protein synthesis, most notably brain-derived neurotrophic factor (BDNF) (Baker-Herman and Mitchell, 2002; Baker-Herman et al., 2004). The subsequent release of BDNF and activation of the high-affinity BDNF receptor tyrosine kinase (TrkB) may strengthen excitatory glutamatergic synapses from brainstem respiratory neurons onto phrenic motoneurons, thus enhancing inspiratory motoneuron activity (Mitchell et al., 2001; Feldman et al., 2003). Here, we hypothesize 
that spinal plasticity induced by intermittent hypoxia has the capacity to augment weak spontaneous recovery of respiratory function after SCI.

Specifically, we tested the hypothesis that intermittent hypoxia, by eliciting pLTF, would strengthen functionally ineffective, crossed-spinal synaptic pathways to phrenic motoneurons, thereby enhancing respiratory recovery below a spinal injury. Indeed, intermittent hypoxia strengthened crossed spinal synaptic pathways by a serotonin-dependent mechanism (i.e., pLTF), although this was not observed at 2 weeks after hemisection. Crossed spinal pLTF was observed at 4 and 8 weeks after injury, and the magnitude of pLTF correlated with injury-induced changes in serotonergic innervation near identified phrenic motoneurons. Because intermittent hypoxia is most effective at enhancing respiratory motor function long after the acute phase of spinal injury, it represents a novel and potentially significant means of enhancing functional recovery after chronic SCI.

\section{Materials and Methods}

Animals. Because rat strain and substrain differences influence the magnitude of respiratory LTF (Fuller et al., 2001b) and the severity of motor and sensory impairment after SCI (Gorman et al., 2001; Mills et al., 2001), the choice of rat strain could influence the ability of intermittent hypoxia to induce pLTF after chronic SCI. To account for this possibility, we studied both inbred Lewis (LW) and outbred Sprague Dawley (SD) rats. Both strains are commonly used in SCI research, and preliminary data suggested differences in the severity of injury caused by cervical hemisection; both strains exhibit a robust pLTF (R. W. Bavis, A. G. Zabka, T. L. Baker-Herman, F. J. Golder, and G. S. Mitchell, unpublished observations). A total of 47 SD (307-400 g; 80-151 d of age; colony 217; Harlan, Indianapolis, IN) and $41 \mathrm{LW}(321-479 \mathrm{~g}$; 98-185 d of age; colony 202B; Harlan) male rats were used in this study. Animals were allocated as control (normal: $\mathrm{SD}, n=8$; LW, $n=8$; sham-operated: $\mathrm{SD}, n=$ 11; LW, $n=8$ ), 2 weeks (SD, $n=10 ; \mathrm{LW}, n=8$ ), 4 weeks (SD, $n=8$; LW, $n=7$ ), and 8 weeks (SD, $n=10 ; \mathrm{LW}, n=10$ ) postinjury groups. Because sex and age-dependent changes in pLTF (Zabka et al., 2001), cervical spinal serotonergic innervation (Ko et al., 1997), and expression of the crossed phrenic phenomenon (Goshgarian, 1979) have been described in rats, age-matched male rats were used in all groups. The initial experiment was designed to investigate crossed-spinal pLTF after intermittent hypoxia in spinally hemisected rats and its serotonin dependence. The second series of experiments explored the relationship between the magnitude of phrenic LTF and recovery of serotonergic innervation near phrenic motoneurons. The Institutional Animal Care and Use Committee at the University of Wisconsin, Madison approved all procedures.

Spinal cord hemisection. Details of the surgical technique have been published previously (Golder et al., 2003). Rats were anesthetized, orotracheally intubated, and mechanically ventilated. After surgical exposure, a 1-mm-long left-sided hemisection was made in the $\mathrm{C} 2$ spinal segment by aspirating spinal tissue through a 23 gauge blunt needle. All animals received postsurgical pain control as described previously. The procedure for sham-operated rats was similar, except the spinal cord remained intact. Rats recovered for 2, 4, or 8 weeks after surgery and were housed alone in standard rodent cages.

Measurement of respiratory motor output. Control and spinally injured rats were initially anesthetized with isoflurane in 50\% oxygen (balance nitrogen) administered via a face mask. A 25 gauge catheter was inserted into the lateral tail vein for drug and fluid administration $(5 \mathrm{ml} / \mathrm{kg} / \mathrm{h}$; 50:50 mixture of lactated Ringer's solution and 6\% hetastarch, with $0.8 \%$ sodium bicarbonate). The trachea was surgically exposed and cannulated, and rats were ventilated throughout the experiment (pump frequency, 55-90 $\mathrm{min}^{-1}$; tidal volume, 2.0-2.5 ml; Rodent Respirator model 683; Harvard Apparatus, South Natick, MA). Both vagus nerves were exposed in the neck and transected to prevent entrainment of respiratory nerve activity with the ventilator. A catheter was positioned in the femoral artery to measure arterial blood pressure (model P122; Grass Telefactor, West Warrick, RI) and sample arterial blood for $\mathrm{pH}$ and blood gas analysis (ABL-500, Radiometer, Copenhagen, Denmark). Endtidal $P_{\mathrm{CO} 2}$ was measured continuously using a mainstream $\mathrm{CO}_{2}$ monitor (Capnoguard; Novametrix Medical Systems, Wallingford, CT). After initial preparation, rats were slowly converted to urethane anesthesia (1.6 $\mathrm{g} / \mathrm{kg}$, i.v.) while isoflurane was discontinued and then paralyzed with pancuronium bromide ( $1 \mathrm{mg} / \mathrm{kg}$, i.v.). Phrenic nerves were isolated via a dorsal approach, desheathed, submerged in mineral oil, and placed on bipolar silver recording electrodes. Nerve activity was amplified (gain, 10,000; A-M Systems, Everett, WA), bandpass-filtered (100 Hz to 10 $\mathrm{kHz}$ ), rectified, and moving averaged (time constant, $50 \mathrm{~ms}$; CWE 821 filter; Paynter, Ardmore, PA). The signal was then digitized, recorded, and analyzed using a WINDAQ data acquisition system (DATAQ Instruments, Akron, $\mathrm{OH}$ ). At least $1 \mathrm{~h}$ elapsed after conversion to urethane anesthesia, thus allowing phrenic neurograms and blood pressure to stabilize.

To establish baseline nerve activity, rats were ventilated with hyperoxia $\left(\mathrm{FI}_{\mathrm{O} 2}, 0.50\right)$, and a level of $\mathrm{Pa}_{\mathrm{CO} 2}$ was established $\sim 3 \mathrm{mmHg}$ above the apneic threshold. Peak integrated, phrenic, nerve-burst amplitude was recorded for $30 \mathrm{~min}$ at baseline conditions, during three 5 min episodes of isocapnic hypoxia $\left(\mathrm{FI}_{\mathrm{O} 2}, 0.10-0.12\right)$ separated by $5 \mathrm{~min}$ isocapnic hyperoxia, and for $60 \mathrm{~min}$ after the last hypoxic episode. Ten rats served as time controls (controls, $n=6$; C2 injured, $n=4$ ) and did not receive intermittent hypoxia; they were maintained under baseline conditions for the duration of a normal protocol. At the end of the experiment, end-tidal $\mathrm{CO}_{2}$ was increased to $90 \mathrm{mmHg}$ for $3 \mathrm{~min}$ to record maximal hypercapnic burst amplitude.

To confirm the role of $5-\mathrm{HT}_{2 \mathrm{~A}}$ receptors in crossed-spinal pLTF (Kinkead and Mitchell, 1999) after cervical hemisection, two Sprague Dawley and two Lewis rats at 8 weeks after injury received a $5-\mathrm{HT}_{2 \mathrm{~A}}$ receptor antagonist (ketanserin, $2 \mathrm{mg} / \mathrm{kg}$, i.v.) $20 \mathrm{~min}$ before the first hypoxic episode.

Arterial blood samples $(0.4 \mathrm{ml}$ in a heparinized glass syringe $)$ were collected for $\mathrm{pH}$ and blood gas measurement before episodic hypoxia, during the first hypoxic episode, and 15, 30, and $60 \mathrm{~min}$ after the last hypoxic episode. Peak moving averaged phrenic neurogram burst amplitude and burst frequency (fictive respiratory rate) were measured off-line over a 30 s period immediately before blood samples. $\mathrm{Pa}_{\mathrm{CO} 2}$ was controlled by adjusting the level of inspired $\mathrm{CO}_{2}$ or by making adjustments in ventilator frequency; blood pressure was maintained by varying the fluid infusion rate. Animals were included in the final analysis only if $\mathrm{Pa}_{\mathrm{O} 2}$ during the hypoxic period was between 35 and $45 \mathrm{mmHg}, \mathrm{Pa}_{\mathrm{O} 2}$ during hyperoxia was above $150 \mathrm{mmHg}$, and $\mathrm{Pa}_{\mathrm{CO} 2}$ during posthypoxia measurement periods was within $1 \mathrm{mmHg}$ of the baseline value.

Immunocytochemistry. Spinal serotonin immunoreactivity was measured in control (normal: $\mathrm{SD}, n=4$; LW, $n=4$; sham-operated: $\mathrm{SD}, n=$ 4; LW $n=4$ ) and spinally injured rats (2 weeks after injury: SD, $n=4$; LW, $n=4$; 4 weeks after injury: SD, $n=4$; LW, $n=4$; 8 weeks after injury: $\mathrm{SD}, n=4$; LW, $n=4$ ) that had been anesthetized with urethane and exposed to intermittent hypoxia. Our goals were to assess the effects of C2 injury on serotonergic innervation of the C4 ventral horn and to allow correlation of crossed-spinal pLTF and serotonin-immunoreactive terminal density. Rats were perfused through the ascending aorta with $300 \mathrm{ml}$ of heparinized saline $(4 \mathrm{IU} / \mathrm{ml}$ ) followed by $500 \mathrm{ml}$ of $4 \%$ paraformaldehyde, $\mathrm{pH} 7.35-7.40$. The cervical spinal cord was removed and stored in $0.1 \mathrm{~m}$ phosphate buffer solution with $0.02 \%$ sodium azide at $4^{\circ} \mathrm{C}$.

Immunocytochemistry was also performed on similar groups of rats (controls: SD, $n=2 ; \mathrm{LW}, n=2 ; 2$ weeks after injury: $\mathrm{SD}, n=2 ; \mathrm{LW}, n=$ $2 ; 8$ weeks after injury: SD, $n=2 ; \mathrm{LW}, n=2$ ) that had not been involved in electrophysiological studies to confirm that observed changes in serotonin terminal density were similar within the (identified) phrenic motor nucleus. These rats were anesthetized using the technique described for spinal hemisection to enable retrograde labeling of phrenic motoneurons. The phrenic nerve on the side of injury was exposed just above the point that the nerve enters the thoracic cavity, and the nerve sheath was injected with the fluorescent tracer Dextran conjugated with Texas Red $(2 \mu \mathrm{l} ; 50 \mathrm{mg} / \mathrm{ml}$; emission spectra, $595 \mathrm{~nm}$; Molecular Probes, Eugene, OR). Rats were allowed to recover for $3 \mathrm{~d}$ and then reanesthetized with 
Table 1. Arterial blood gases, arterial blood pH, phrenic burst frequency (fictive respiratory rate), and mean arterial blood pressure measurements during baseline conditions, hypoxia, and $60 \mathrm{~min}$ after IH in control rats and $\mathrm{C}_{2}$-injured rats at 2, 4, and 8 weeks after injury for Sprague Dawley and Lewis rats

\begin{tabular}{|c|c|c|c|c|c|c|c|}
\hline & & \multicolumn{3}{|l|}{ Sprague Dawley } & \multicolumn{3}{|l|}{ Lewis } \\
\hline & & Baseline & Нypoxia & 60 min after IH & Baseline & Нypoxia & 60 min after IH \\
\hline \multirow[t]{4}{*}{$\mathrm{pH}$} & Control & $7.39 \pm 0.01^{a}$ & $7.36 \pm 0.01$ & $7.38 \pm 0.01^{a}$ & $7.38 \pm 0.01^{a}$ & $7.35 \pm 0.01$ & $7.38 \pm 0.01^{a}$ \\
\hline & 2 weeks & $7.43 \pm 0.02^{a, d}$ & $7.39 \pm 0.03^{d}$ & $7.44 \pm 0.01^{a_{\prime}^{d}}$ & $7.40 \pm 0.01^{a, d}$ & $7.40 \pm 0.01^{d}$ & $7.42 \pm 0.01^{a, d}$ \\
\hline & 4 weeks & $7.40 \pm 0.01^{a, d, e}$ & $7.35 \pm 0.01^{d, e}$ & $7.38 \pm 0.01^{a, d, e}$ & $7.41 \pm 0.02^{a, d, e}$ & $7.40 \pm 0.02^{d, e}$ & $7.40 \pm 0.02^{a, d, e}$ \\
\hline & 8 weeks & $7.41 \pm 0.01^{a, d, e}$ & $7.39 \pm 0.01^{d, e}$ & $7.40 \pm 0.01^{a, d, e}$ & $7.38 \pm 0.01^{a, d, e}$ & $7.38 \pm 0.01^{d, e}$ & $7.38 \pm 0.01^{a, d, e}$ \\
\hline \multirow[t]{4}{*}{$\mathrm{PaCO}_{2}(\mathrm{mmHg})$} & Control & $37 \pm 1$ & $37 \pm 1$ & $37 \pm 1$ & $42 \pm 1^{b}$ & $41 \pm 1^{b}$ & $42 \pm 1^{b}$ \\
\hline & 2 weeks & $33 \pm 1$ & $34 \pm 2$ & $34 \pm 1$ & $40 \pm 2^{b}$ & $40 \pm 2^{b}$ & $40 \pm 2^{b}$ \\
\hline & 4 weeks & $37 \pm 2$ & $38 \pm 2$ & $37 \pm 1$ & $40 \pm 1^{b}$ & $41 \pm 1^{b}$ & $40 \pm 1^{b}$ \\
\hline & 8 weeks & $36 \pm 1$ & $37 \pm 1$ & $36 \pm 1$ & $40 \pm 1^{b}$ & $40 \pm 1^{b}$ & $40 \pm 1^{b}$ \\
\hline \multirow[t]{4}{*}{$\mathrm{PaO}_{2}(\mathrm{mmHg})$} & Control & $275 \pm 6^{a}$ & $38 \pm 1$ & $261 \pm 6^{a, c}$ & $229 \pm 19^{a, b}$ & $40 \pm 1$ & $240 \pm 11^{a}$ \\
\hline & 2 weeks & $290 \pm 7^{a}$ & $39 \pm 1$ & $276 \pm 4^{a}$ & $267 \pm 20^{a}$ & $42 \pm 1^{b}$ & $254 \pm 17^{a}$ \\
\hline & 4 weeks & $270 \pm 13^{a}$ & $38 \pm 2$ & $258 \pm 12^{a}$ & $240 \pm 18^{a}$ & $39 \pm 1$ & $231 \pm 16^{a}$ \\
\hline & 8 weeks & $267 \pm 7^{a}$ & $39 \pm 2$ & $261 \pm 7^{a}$ & $235 \pm 19^{a}$ & $40 \pm 2$ & $203 \pm 23^{a, c}$ \\
\hline \multirow[t]{4}{*}{$\mathrm{MBP}(\mathrm{mmHg})$} & Control & $128 \pm 5^{a}$ & $97 \pm 5$ & $106 \pm 4^{a, c}$ & $70 \pm 3^{a, b}$ & $46 \pm 3^{b}$ & $55 \pm 3^{b, c}$ \\
\hline & 2 weeks & $100 \pm 11^{a, d}$ & $54 \pm 6^{d}$ & $78 \pm 5^{a, c, d}$ & $82 \pm 6^{a}$ & $58 \pm 5$ & $56 \pm 3^{b, c}$ \\
\hline & 4 weeks & $130 \pm 10^{a, e}$ & $94 \pm 13^{e}$ & $106 \pm 9^{a, c, e}$ & $75 \pm 11^{a, b}$ & $49 \pm 8^{b}$ & $52 \pm 5^{b, c}$ \\
\hline & 8 weeks & $105 \pm 3^{a, d, f}$ & $68 \pm 6^{d, f}$ & $74 \pm 5^{a, c, d, f}$ & $83 \pm 5^{a}$ & $59 \pm 6$ & $56 \pm 5^{b, c}$ \\
\hline \multirow[t]{4}{*}{$\mathrm{RR}\left(\min ^{-1}\right)$} & Control & $41 \pm 2^{a}$ & $59 \pm 2$ & $45 \pm 1^{a, c}$ & $40 \pm 1^{a}$ & $42 \pm 2$ & $44 \pm 1^{a, c}$ \\
\hline & 2 weeks & $43 \pm 2^{a, d}$ & $57 \pm 2$ & $45 \pm 3^{a, c, d}$ & $48 \pm 1^{a, d}$ & $51 \pm 1^{d}$ & $48 \pm 1^{a, c, d}$ \\
\hline & 4 weeks & $47 \pm 2^{a, d}$ & $57 \pm 2$ & $48 \pm 2^{a, c, d}$ & $46 \pm 1^{a, d}$ & $51 \pm 2^{d}$ & $50 \pm 2^{a, c, d}$ \\
\hline & 8 weeks & $51 \pm 3^{a, d, e, f}$ & $64 \pm 3$ & $52 \pm 3^{a, c, d, e, f}$ & $53 \pm 2^{a, d, e, f}$ & $54 \pm 2^{d}$ & $53 \pm 1^{a, c, d, e, f}$ \\
\hline
\end{tabular}

Spinal injury increased arterial pH in both strains, and this effect was greatest at 2 weeks after injury $(p<0.05)$. Arterial $P_{\text {co2 }}$ was not altered by injury, suggesting the presence of a nonrespiratory alkalosis after spinal injury. LW rats had higher $\mathrm{Pa}_{\mathrm{CO} 2}$ throughout the protocol compared with SD rats $(p<0.05)$. ( 2 hemisection decreased mean arterial blood pressure in SD rats at 2 and 8 weeks but not 4 weeks after injury $(p<0.05)$. Spinal injury did not change blood pressure in LW rats. Blood pressure was lower in LW than SD rats in the control and 4 weeks postinjury groups $(p<0.05)$. IH, Intermittent hypoxia; MBP, mean arterial blood pressure; RR, respiratory rate.

${ }^{a}$ Different from the hypoxic value.

${ }^{b}$ Different from Sprague Dawley.

'Different from the baseline value.

${ }^{d}$ Different from control.

${ }^{e}$ Different from 2 weeks after injury.

fDifferent from 4 weeks after injury.

sodium pentobarbital (120 mg/kg, i.p.) to allow perfusion as described above.

The C4 spinal segment was cryoprotected with $20 \%$ sucrose and $5 \%$ glycerol in $0.1 \mathrm{M}$ PBS, and $50 \mu \mathrm{m}$ transverse sections were cut with a freezing microtome. Four representative sections were selected for each animal. Sections were washed, incubated in 3\% hydrogen peroxide for 30 min, washed, and incubated in $1 \%$ sodium borohydride for $30 \mathrm{~min}$. Sections were blocked with $3 \%$ normal goat serum for $30 \mathrm{~min}$ and incubated overnight in primary serotonin antibody made in rabbit (1:5000) with $1 \%$ normal goat serum and $0.75 \%$ Triton X-100. Details of primary antisera production, isolation, and characterization were as reported previously (Brownfield et al., 1998). Sections were then washed, incubated in goat anti-rabbit IgG (1:100; Vector Laboratories, Burlingame, $\mathrm{CA})$, washed, and then incubated in peroxidase anti-peroxidase (1:800; Sigma, St. Louis, MO). Finally, sections were reacted with $0.04 \% 3,3^{\prime}$ diaminobenzidine, mounted, dehydrated, and coverslipped.

Image analysis. Light microscopy images of the $\mathrm{C} 4$ ventral horn on both sides of the cord were captured at $20 \times$ and $40 \times$ magnification with a digital camera (SPOT II; Diagnostics Instruments, Sterling Heights, MI). Sections from rats with back-labeled phrenic motoneurons were examined under fluorescent microscopy to position the field over the phrenic motor nucleus. SPOT software (version 3.5.1) and Adobe Photoshop (version 7.0; Adobe Systems, San Jose, CA) were used for digital image processing. Equivalent adjustments to tone scale, gamma, and sharpness were made across all images. Sections with back-labeled motoneurons were collated in Adobe Photoshop as overlapping bright-field images and fluorescent-field images to create one image.

Serotonin immunoreactivity was measured using imaging software (Image-Pro Plus, version 4.5.1.22; Media Cybernetics, Silver Spring, MD). A circular area-of interest was positioned over the ventral horn on both sides of the spinal cord. For rats without back-labeled motoneurons, area of interest, size, and position was standardized across sections by choosing a circular circumference that matched the curvature of the ventral horn at $20 \times$ magnification. The size and position of the area of interest encompassed the approximate location of the phrenic nucleus based on preliminary retrograde labeling studies of phrenic motoneurons. The area of interest for images with back-labeled motoneurons was centered over the phrenic nucleus at $40 \times$ magnification. Using the imaging software, a histogram-based color range was chosen that selectively identified serotonin-positive elements in the area of interest. The number of pixels occupied by serotonin-positive terminals was quantified within the area of interest.

Statistical analysis. Burst frequency and peak amplitude were averaged over $30 \mathrm{~s}$ periods immediately before blood sampling for blood gas measurements. Baseline burst amplitude and changes in amplitude during and after hypoxia are reported as absolute values (volts) and expressed as a percentage of hypercapnic maximal amplitude. Burst frequency is reported as absolute values and as the change from the baseline value. Serotonin immunoreactivity was averaged over four sections per animal and then averaged when grouped by time after injury.

Normal and sham-operated rats of each strain were similar with respect to blood pressure, blood gases, and phrenic activity during baseline, hypoxic, and posthypoxic conditions at 2, 4, and 8 weeks after injury (Table 1). In addition, the distribution of serotonin-containing elements throughout the ventral horn in normal and sham-operated rats was similar to a previous study (Steinbusch, 1981). Thus, normal and shamoperated rats were combined into a single control group for subsequent statistical analyses. Assumptions of normality and equal variance were tested before parametric analyses were performed. When these assumptions could not be confirmed, nonparametric analysis was performed with Kruskal-Wallis ANOVAs followed by Mann-Whitney $U$ tests. Otherwise, statistical inferences were based on a parametric two-way ANOVA (factors, time postinjury and strain), with individual comparisons made based on the Student-Neuman-Keuls post hoc test. Differences were considered significant if $p<0.05$. All values are expressed as mean \pm SEM. 

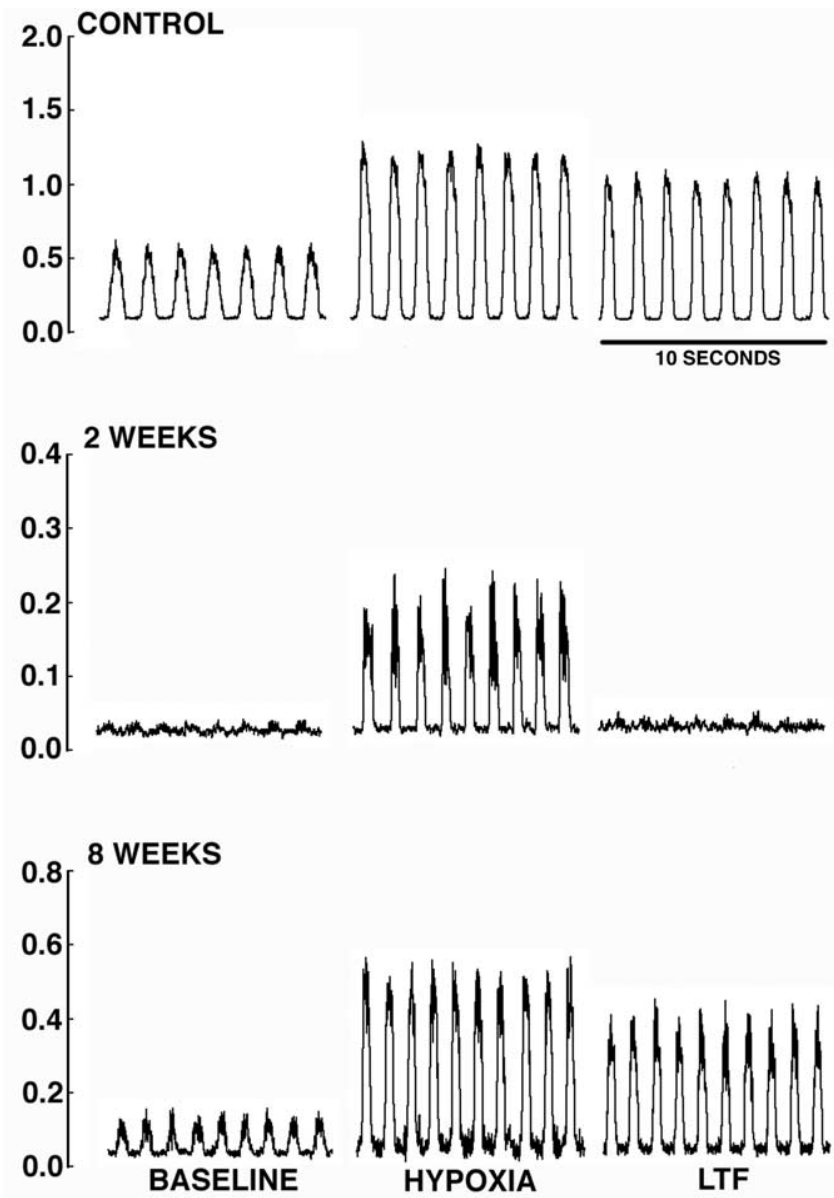

Figure 1. Representative phrenic neurograms on the side of injury from control and spinally injured rats at 2 and 8 weeks after injury during baseline conditions, hypoxia, and 60 min after hypoxia (LTF). Spinally injured rats show partial recovery of baseline phrenic burst amplitude (volts) that increases progressively with time after injury (note different $y$-axis scales). Phrenic burst frequency also progressively increases after $\mathrm{SCl}$. All rats show a robust phrenic amplitude response to hypoxia. pLTF is absent at 2 weeks but recovers by 8 weeks after injury.

\section{Results}

Spontaneous phrenic motor recovery

Over a period of 8 weeks, spontaneous phrenic motor recovery was visible and audible in the phrenic neurogram ipsilateral to cervical hemisection, and these small bursts of activity were synchronous with phrenic bursts on the uninjured side. The amplitude of respiratory bursts increased during hypoxia. Thus, the (spontaneous) crossed-phrenic phenomenon was consistent with previous reports (Nantwi et al., 1999; Golder et al., 2001; Fuller et al., 2003). On the side of injury, respiratory bursts (as absolute and normalized amplitude) were smaller than on the contralateral side and versus control rats. However, there was a weak time-dependent increase in activity between 2 and 8 weeks (Figs. 1, 2) $(p<0.05)$. The recovery of nerve burst amplitude was accompanied by a progressively increasing respiratory rate (Table $1 ; p<0.05)$.

\section{Phrenic long-term facilitation}

Short-term phrenic responses to intermittent hypoxia were robust in all rats and returned to baseline activity after each hypoxic episode (Figs. 1, 2). In control rats, phrenic burst amplitude then progressively increased over the ensuing hour, revealing pLTF [SD, $16 \pm 2 \%$; LW, $28 \pm 3 \%$ of maximal hypercapnic amplitude] (Figs. 1, 2). In contrast, no crossed-spinal pLTF was observed
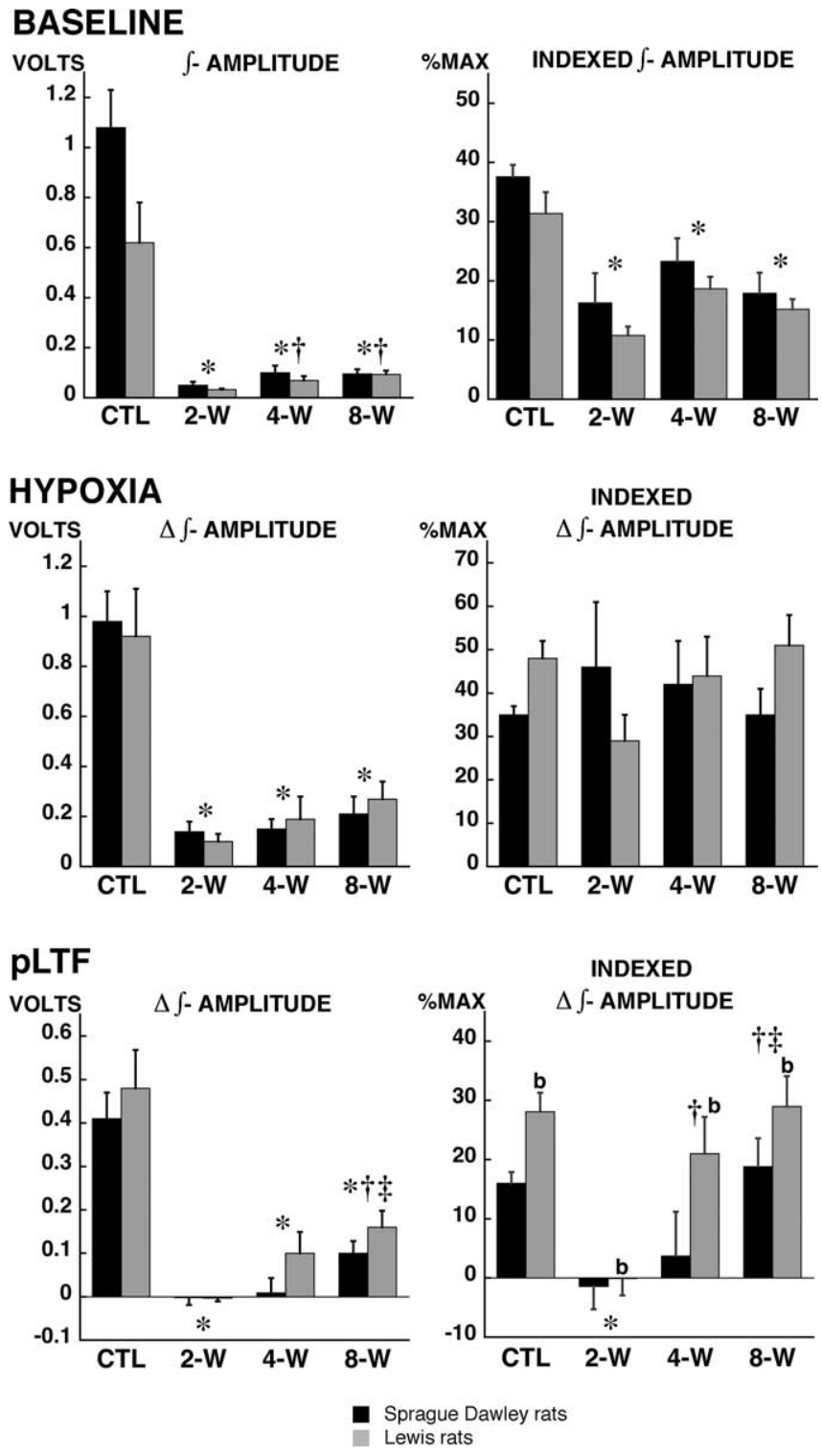

Figure 2. Inspiratory phrenic nerve activity during baseline, hypoxic, and posthypoxic (pLTF) conditions in control (CTL) and spinally injured rats at 2 weeks $(2-W), 4$ weeks $(4-W)$, and 8 weeks (8-W) after injury. SCI decreased baseline moving-averaged $\left(\int\right)$ phrenic burst amplitude and amplitude indexed to hypercapnic maximal amplitude (\%MAX). Hemisection also decreased the change in moving-averaged $\left(\int\right)$ phrenic burst amplitude as absolute values but not when indexed to hypercapnic maximal amplitude (\%MAX). Finally, SCI minimized crossedspinal pLTF at 2 weeks after injury; pLTF recovered by 8 weeks after injury. pLTF was larger in LW versus SD rats. * , Different from controls; $\uparrow$, different from 2 weeks after injury; $b$, different from SD rats.

after intermittent hypoxia at 2 weeks after injury (SD, $-1.4 \pm$ $4 \%$; LW,$-0.1 \pm 3 \%$ ) (Figs. 1, 2) despite the presence of pLTF on the uninjured side (SD, $12 \pm 5 \%$; LW, $13 \pm 4 \%$ ). At 4 weeks after injury, crossed-spinal pLTF was observed in Lewis rats; however, a small pLTF in Sprague Dawley rats was not different from time controls (SD, $4 \pm 7 \%$, NS; LW, $21 \pm 6 \%$; $p<0.05$ ) (Figs. 1, 2). At 8 weeks after injury, crossed-spinal pLTF was evident and comparable in both strains; normalized pLTF had increased and was now comparable with values found in control (uninjured) rats (SD, $19 \pm 5 \%$; LW, $29 \pm 5 \%$ ) (Figs. 1, 2) (both, $p<0.05$ ). In absolute terms, as expressed by the voltage change in phrenic burst amplitude, crossed-spinal pLTF remained smaller than in control animals $(p<0.05)$. Thus, intermittent hypoxia aug- 

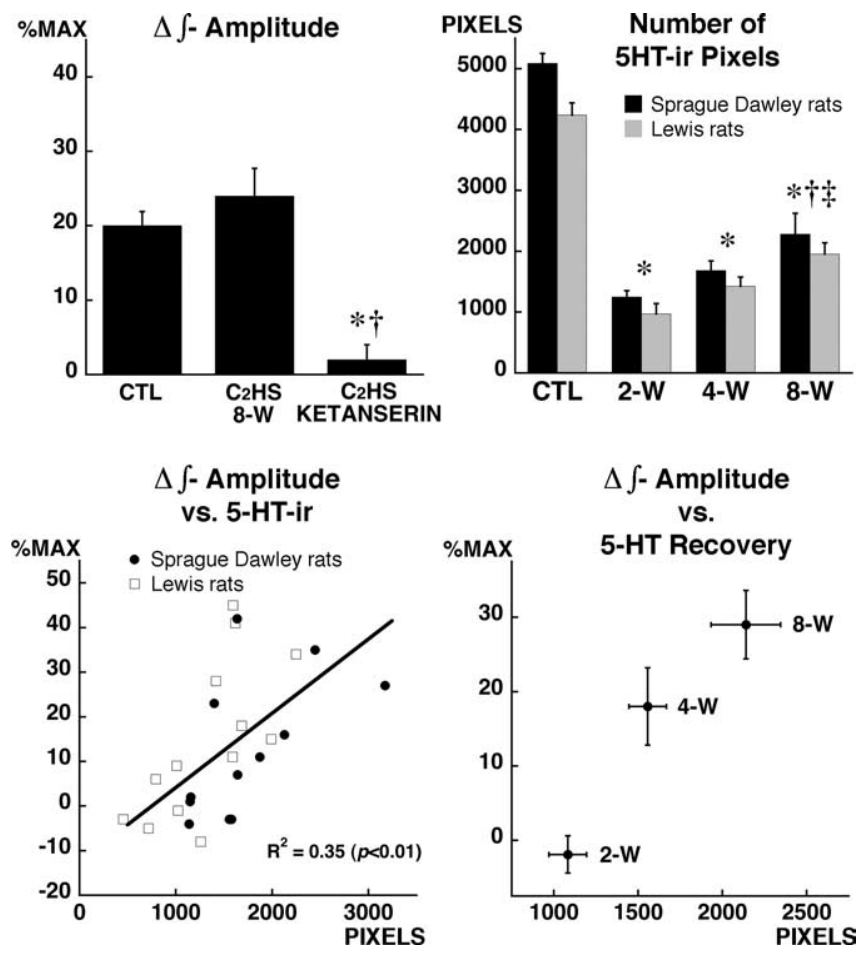

Figure 3. Serotonin immunoreactivity (5HT-ir) correlates with phrenic LTF on the side of injury in control (CTL) and spinally injured (C2HS) rats at 2 weeks (2-W), 4 weeks (4-W), and 8 weeks (8-W) after injury. Ketanserin (KTSRN), a 5- $\mathrm{HT}_{2 \mathrm{~A}}$ receptor antagonist, abolished LTF at 8 weeks after injury. The magnitude of pLTF on the side of injury was positively correlated to the number of pixels occupied by serotonin-positive terminals in the ( 4 ventral horn $(p<0.05)$. *, Different from controls; $\dagger$, different from 2 weeks after injury; $\neq$, different from 8 weeks after injury.

ments functional recovery of respiratory activity in rats with a chronic spinal injury by eliciting crossed-spinal pLTF but only after $4-8$ postinjury weeks.

\section{Serotonin receptor antagonist (ketanserin)}

To confirm the serotonin-dependence of crossed-spinal pLTF after cervical hemisection, two Sprague Dawley and two Lewis rats at 8 weeks after injury were pretreated with ketanserin, a $5-\mathrm{HT}_{2 \mathrm{~A}}$ receptor antagonist known to block pLTF in uninjured rats (Kinkead and Mitchell, 1999; Fuller et al., 2001a). Ketanserin blocked pLTF on both sides of the injured spinal cord (Fig. 3) ( $p<0.05$; uninjured side) (data not shown), confirming that crossed-spinal pLTF after cervical hemisection is both serotonin dependent and requires $5-\mathrm{HT}_{2 \mathrm{~A}}$ receptor activation.

Serotonergic innervation near the phrenic motor nucleus after C2 hemisection

To examine the potential role of serotonergic innervation below the spinal injury in restoring crossed-spinal pLTF, we evaluated the effects of injury and time on serotonergic innervation near phrenic motoneurons. The magnitude of pLTF was strongly correlated with serotonin immunoreactivity in $\mathrm{C} 4$ ventral horn of the same rat. Thus, serotonin immunoreactivity on the injured side was almost completely absent in the $\mathrm{C} 4$ ventral horn (Fig. 3 ) $(p<0.05)$ and phrenic motor nucleus (Fig. 4$)(p<0.05)$ at 2 weeks after injury, consistent with a previous study (Golder et al., 2001). A smaller decrease was also observed on the uninjured side (controls: SD, $5090 \pm 159$; LW, $4235 \pm 202 ; 2$ weeks: SD, $2759 \pm$ 110; LW, $2422 \pm 437$; number of pixels; $p<0.05$ ). At 8 weeks after injury, the collective area of serotonin-positive terminals on the injured side had partially recovered in the $\mathrm{C} 4$ ventral horn
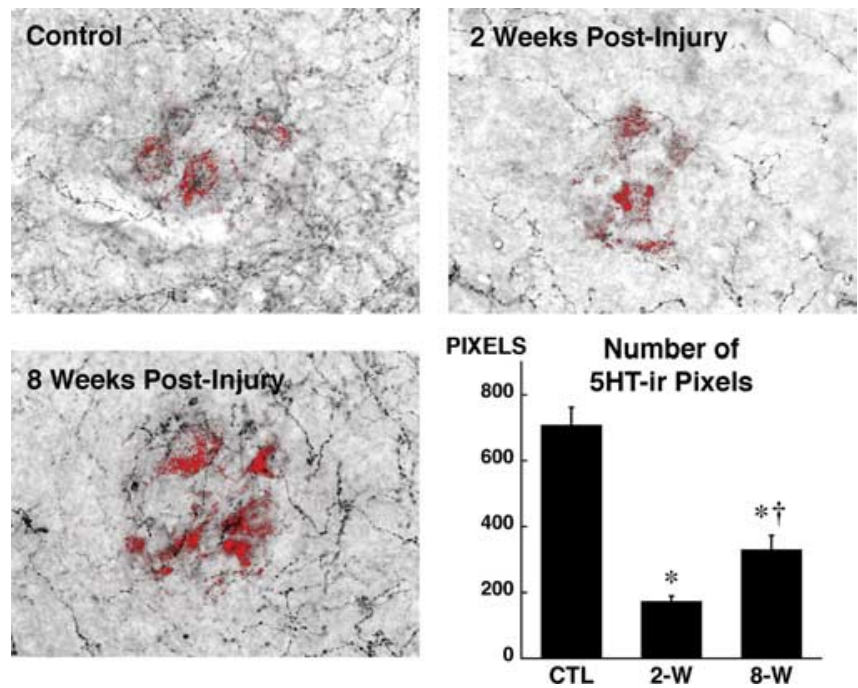

Figure 4. Photomicrographs of and recovery in serotonergic innervation near labeled phrenic motoneurons in control (CTL) and spinally injured rats at 2 weeks (2-W) and 8 weeks (8-W) after injury. Phrenic motoneurons (red) were back-labeled with Dextran conjugated to Texas Red and visualized with fluorescent microscopy. ${ }^{*}$, Different from controls; $\uparrow$, different from 2 weeks after injury.

(Fig. 3) $(p<0.05)$ and phrenic motor nucleus (Fig. 4) $(p<$ $0.05)$. Recovery on the uninjured side was more complete (controls: SD, $5090 \pm 159$; LW, $4235 \pm 202$; 8 weeks: SD, $4290 \pm 260$; LW, $3630 \pm 258$; number of pixels). Thus, after descending serotonergic inputs are disrupted by hemisection, serotonergic innervation partially recovers over an 8 weeks period, most likely because of sprouting in the projections of surviving crossed-spinal serotonergic fibers.

Regression analysis of pLTF with serotonergic terminal area in the same rats revealed a strong positive correlation (Fig. 3) $(p<$ 0.05 ). When $\mathrm{C} 2$-injured rats were grouped by time after injury, the magnitude of crossed-spinal pLTF also was positively correlated with recovery of serotonergic innervation below the injury (Fig. 3) $(p<0.05)$. No similar relationships were identified between the magnitude of pLTF and serotonergic innervation on the uninjured side (data not shown).

\section{Strain interactions}

During hypoxic episodes, there were no differences in phrenic minute activity between strains. However, Lewis rats responded to hypoxia with a greater change in amplitude and smaller change in respiratory frequency relative to Sprague Dawley rats (Fig. 2, Table 1) $(p<0.05)$. After intermittent hypoxia, the magnitude of crossed spinal pLTF was greater in Lewis versus Sprague Dawley rats (Fig. 2) $(p<0.05)$, and recovery of pLTF occurred more rapidly ( 4 weeks in Lewis and 8 weeks in Sprague Dawley rats).

\section{Discussion}

Acute intermittent hypoxia enhances functional recovery of respiratory motor output below a chronic SCI by activating serotonin-dependent neuroplasticity. However, intermittent hypoxia is not effective within 2 weeks of injury and becomes effective only 4-8 weeks after injury. Time-dependent pLTF below the spinal hemisection is strongly correlated with the initial loss and subsequent partial recovery of serotonergic innervation near phrenic motoneurons. Because it is most effective in chronically injured rats, intermittent hypoxia may hold promise as a means of augmenting functional recovery beyond the acute phase of SCI. Thus, intermittent hypoxia (or related interventions) may 
convert functionally ineffective synaptic pathways to effective pathways, thereby restoring respiratory motor function at a time when the prognosis for additional recovery is minimal.

Intermittent hypoxia represents a means to harness spinal neuroplasticity, potentially improving spinal function without physical activity. Quadriplegics are unable to exercise appreciably, and, unlike intensive physical therapy, acute intermittent hypoxia is easy to apply and carries minimal risk, because only a small number of mild to moderate hypoxic episodes are required for an enduring effect on respiratory motor output (Powell and Garcia, 2000; Serebrovskaya, 2002). Furthermore, because of progress made toward understanding the cellular mechanisms of pLTF (Baker-Herman et al., 2004; Bocchiaro and Feldman, 2004), it may be possible to intervene downstream from intermittent hypoxia and serotonin release, bypassing practical limits in the use of intermittent hypoxia during the acute phase of SCI. Because more robust protocols of intermittent hypoxia may cause morbidity such as neurocognitive deficits (Row et al., 2003), the potential benefits of further strengthening spinal synaptic pathways to respiratory motoneurons (Fuller et al., 2003) may be offset.

The duration of pLTF after intermittent hypoxia is unknown. Normally, pLTF lasts for a minimum of 90 min after three hypoxic episodes in anesthetized rats. However, after 10 hypoxic episodes per day for $7 \mathrm{~d}$, enhanced respiratory LTF is observed up to $24 \mathrm{~h}$ later (J. R. Wilkerson and Mitchell, unpublished observations). After chronic intermittent hypoxia (12 h per night for 7 nights), crossed spinal synaptic pathways to phrenic motoneurons are enhanced even at 2 weeks after hemisection (Fuller et al., 2003). Chronic intermittent hypoxia exerts prolonged effects on respiratory motor output that last for at least 1 week (Ling et al., 2001). If pLTF after acute intermittent hypoxia is of limited duration, intermittent hypoxia may still exert beneficial effects. For example, short-term increases in synaptic strength may facilitate physical therapy targeting either further strengthening synaptic pathways to respiratory motoneurons or respiratory muscle strength.

\section{Strengthening silent synaptic pathways to phrenic motoneurons} Weak recovery of phrenic (and diaphragmatic) inspiratory activity occurs spontaneously with time after C2 hemisection (Nantwi et al., 1999; Golder et al., 2001, 2003; Fuller et al., 2003). Acute intermittent hypoxia augmented respiratory output on the side of injury, possibly by accelerating or enhancing the same mechanism of the spontaneous crossed-phrenic phenomenon. In contrast, intermittent hypoxia may enhance crossed spinal synaptic pathways by distinct mechanisms. C2 hemisection removes all descending premotor drive to ipsilateral phrenic motoneurons but spares a small population of premotor axons that cross the spinal midline caudal to injury (Moreno et al., 1992; Goshgarian et al., 1993). These crossed spinal pathways are normally silent but become functional within days or weeks after injury (Nantwi et al., 1999; Golder et al., 2001, 2003; Fuller et al., 2003). Thus, pLTF on the side of injury reflects an augmentation of existing, but functionally ineffective, synaptic pathways.

Considerable progress has been made in developing a cellular/ synaptic model of pLTF in spinally intact rats (Mitchell et al., 2001; Feldman et al., 2003; Baker-Herman et al., 2004; Bocchiaro and Feldman, 2004). pLTF is elicited by intermittent, but not sustained, hypoxia (Baker and Mitchell, 2000), requires $\left(5-\mathrm{HT}_{2 \mathrm{~A}}\right.$ ) serotonin receptor activation within the region of the phrenic motor nucleus (Fuller et al., 2001a; Baker-Herman and Mitchell, 2002), is spinal protein-synthesis dependent (Baker-Herman and
Mitchell, 2002), and requires the synthesis and release of new BDNF protein (Baker-Herman et al., 2004). In our working model of pLTF, serotonin-dependent BDNF synthesis and release activate presynaptic and/or postsynaptic TrkB receptors and strengthen glutamatergic synapses between descending respiratory neurons and phrenic motoneurons (Fuller et al., 2000; Feldman et al., 2003; Bocchiaro and Feldman, 2004). Similar mechanisms most likely underlie crossed-spinal pLTF in C2hemisected rats, because $5-\mathrm{HT}_{2 \mathrm{~A}}$ receptor activation is still required.

Transformation of silent to effective synaptic pathways by serotonin and BDNF has been described in the spinal dorsal horn (Itami et al., 2003; Kim et al., 2003). Presynaptically, serotonin induces synaptic vesicle filling of preexisting empty varicosities and permits growth of new functional varicosities (Kim et al., 2003). Postsynaptically, BDNF and serotonin can strengthen silent synapses by increased insertion of cytosolic AMPA receptors in the postsynaptic membrane (Zhuo, 2000; Itami et al., 2003). Given the serotonin dependence of pLTF, the inability to elicit pLTF 2 weeks after C2 spinal hemisection is predictable, because descending ipsilateral projections from brainstem serotonergic neurons are essentially disrupted. However, the partial restoration of serotonergic innervation within the phrenic motor nucleus (Figs. 3, 4), possibly combined with increased expression of 5- $\mathrm{HT}_{2 \mathrm{~A}}$ receptors (Fuller et al., 2005), reestablishes conditions necessary for pLTF expression.

The critical role for serotoninergic terminals in crossed spinal pLTF is illustrated by the following results: (1) the magnitude of pLTF was correlated with the initial loss and then recovery of serotonergic innervation near phrenic motoneurons, and (2) ketanserin abolished pLTF on both sides of the injured spinal cord 8 weeks after injury. The correlation between pLTF and serotonin terminal density suggests, but does not confirm, causality. The ketanserin sensitivity of pLTF below a spinal hemisection increases confidence that crossed-spinal pLTF posthemisection results from the same serotonin-dependent mechanism that underlies pLTF in intact rats. On balance, the evidence strongly suggests that the restoration of spinal serotonergic innervation underlies, at least in part, the recovery of pLTF after hemisection.

\section{Serotonin and motor recovery after SCI}

Serotonin has been implicated in other forms of functional recovery after chronic SCI. For example, a serotonergic neurotoxin abolishes functional recovery of locomotion after experimental SCI in rats (Hashimoto and Fukuda, 1991). In addition, loss of serotonergic innervation of the spinal dorsal horn is associated with onset of chronic pain (Hains et al., 2002); consequently, restoration of serotonergic function restores more normal nociceptive processing (Hains et al., 2001a,b, 2003). Similarly, a serotonergic neurotoxin abolished the spontaneous crossed phrenic phenomenon in rats with chronic spinal hemisection (Golder et al., 2001), and serotonin depletion abolished the crossed phrenic phenomenon evoked by asphyxia shortly after C2 hemisection (Hadley et al., 1999). Collectively, these studies suggest a critical role for endogenous serotonin in activating silent crossed-spinal synaptic pathways.

Locomotor recovery in spinally injured rats is correlated with the return of serotonin-positive terminals to the ipsilateral lumbosacral ventral horn (Saruhashi et al., 1996). In contrast, neither phrenic motor recovery nor serotonergic innervation of the phrenic motor nucleus was complete at any time examined, either before or after acute intermittent hypoxia. Differential recovery among motor systems may be more apparent than real, 
because locomotion is more difficult to quantify than respiratory activity. Alternately, there may be real differences between motor systems, such as preservation of the locomotor central pattern generator below the site of injury (vs disruption of pathways from the supraspinal respiratory pattern generator) (Feldman et al., 2003).

\section{Acute versus chronic intermittent hypoxia and phrenic motor function after SCI}

Chronic intermittent hypoxia (i.e., 7 nights of episodic hypoxia) induces multiple forms of respiratory plasticity, including enhanced pLTF (Ling et al., 2001; Mitchell et al., 2001). Enhanced pLTF after chronic intermittent hypoxia in spinally intact rats requires the activation of both $5-\mathrm{HT}_{2 \mathrm{~A}}$ and non-5- $\mathrm{HT}_{2 \mathrm{~A}}$ receptors (Ling et al., 2001), probably of the $5-\mathrm{HT}_{4}, 5-\mathrm{HT}_{6}$, or $5-\mathrm{HT}_{7}$ receptor subtype (McGuire et al., 2004). Chronic intermittent hypoxia also strengthens crossed spinal synaptic pathways to phrenic motoneurons in rats with chronic C2 hemisection, although not in spinally intact rats (Fuller et al., 2003). The utility of chronic intermittent hypoxia to enhance synaptic pathways to phrenic motoneurons may be offset by the potential for deleterious side effects such as altered sympathetic chemoreflexes (Greenberg et al., 1999), hippocampal cell death (Gozal et al., 2001), or neurocognitive deficits (Row et al., 2002).

\section{Functional significance}

Although most SCIs are incomplete, spontaneous functional recovery occurs in many motor systems via spared pathways (Raineteau and Schwab, 2001), including breathing (Goshgarian, 2003). Yet endogenous recovery is often slow in onset and inadequate to restore normal function. Unfortunately, few therapeutic options are available after the acute, postinjury period, when mechanisms of spontaneous recovery are exhausted and additional functional improvement is unlikely (McDonald et al., 2002). Intermittent hypoxia has interesting implications as a possible therapeutic approach to humans with chronic cervical SCI. For example, after SCI, it may be that respiratory function along residual neural pathways can be enhanced after a relatively innocuous respiratory maneuver, and that physical therapy aimed at strengthening respiratory muscle performance (Gutierrez et al., 2003) may be facilitated by previous administration of intermittent hypoxia to strengthen residual synaptic pathways.

\section{References}

Baker TL, Mitchell GS (2000) Episodic but not continuous hypoxia elicits long-term facilitation of phrenic motor output in rats. J Physiol (Lond) 529:215-219.

Baker-Herman TL, Mitchell GS (2002) Phrenic long-term facilitation requires spinal serotonin receptor activation and protein synthesis. J Neurosci 22:6239-6246.

Baker-Herman TL, Fuller DD, Bavis RW, Zabka AG, Golder FJ, Doperalski NJ, Johnson RA, Watters JJ, Mitchell GS (2004) BDNF is necessary and sufficient for spinal respiratory plasticity following intermittent hypoxia. Nat Neurosci 7:48-55.

Blight AR (2004) Just one word: plasticity. Nat Neurosci 7:206-208.

Bocchiaro CM, Feldman JL (2004) Synaptic activity-independent persistent plasticity in endogenously active mammalian motoneurons. Proc Natl Acad Sci USA 101:4292-4295.

Brownfield MS, Yracheta J, Chu F, Lorenz D, Diaz A (1998) Functional chemical neuroanatomy of serotonergic neurons and their targets: antibody production and immunohistochemistry (IHC) for 5 - $\mathrm{HT}$, its precursor $\left(5-\mathrm{HT}_{\mathrm{P}}\right)$ and metabolite (5-HIAA), biosynthetic enzyme (TPH), transporter (SERT), and three receptors $\left(5-\mathrm{HT}_{2 \mathrm{~A}}, 5-\mathrm{HT}_{5 \mathrm{~A}}, 5-\mathrm{HT}_{7}\right)$. Ann NY Acad Sci 861:232-233.

Feldman JL, Mitchell GS, Nattie EE (2003) Breathing: rhythmicity, plasticity, chemosensitivity. Annu Rev Neurosci 26:239-266.
Fuller DD, Bach KB, Baker TL, Kinkead R, Mitchell GS (2000) Long term facilitation of phrenic motor output. Respir Physiol 121:135-146.

Fuller DD, Zabka AG, Baker TL, Mitchell GS (2001a) Phrenic long-term facilitation requires 5-HT receptor activation during but not following episodic hypoxia. J Appl Physiol 90:2001-2006.

Fuller DD, Baker TL, Behan M, Mitchell GS (2001b) Expression of hypoglossal long-term facilitation differs between substrains of SpragueDawley rat. Physiol Genomics 4:175-181.

Fuller DD, Johnson SM, Olson Jr EB, Mitchell GS (2003) Synaptic pathways to phrenic motoneurons are enhanced by chronic intermittent hypoxia after cervical spinal cord injury. J Neurosci 23:2993-3000.

Fuller DD, Baker-Herman TL, Golder FJ, Doperalski NJ, Watters JJ, Mitchell GS (2005) Cervical spinal cord injury upregulates ventral spinal 5-HT ${ }^{2 \mathrm{~A}}$ receptors. J Neurotrauma 22:203-213.

Golder FJ, Reier PJ, Bolser DC (2001) Altered respiratory motor drive after spinal cord injury: supraspinal and bilateral effects of a unilateral lesion. J Neurosci 21:8680-8689.

Golder FJ, Fuller DD, Davenport PW, Johnson RD, Reier PJ, Bolser DC (2003) Respiratory motor recovery after unilateral spinal cord injury: eliminating crossed phrenic activity decreases tidal volume and increases contralateral respiratory motor output. J Neurosci 23:2494-2501.

Gorman AL, Yu CG, Ruenes GR, Daniels L, Yezierski RP (2001) Conditions affecting the onset, severity, and progression of a spontaneous pain-like behavior after excitotoxic spinal cord injury. J Pain 2:229-240.

Goshgarian HG (1979) Developmental plasticity in the respiratory pathway of the adult rat. Exp Neurol 66:547-555.

Goshgarian HG (2003) The crossed phrenic phenomenon: a model for plasticity in the respiratory pathways following spinal cord injury. J Appl Physiol 94:795-810.

Goshgarian HG, Ellenberger HH, Feldman JL (1993) Bulbospinal respiratory neurons are a source of double synapses onto phrenic motoneurons following cervical spinal cord hemisection in adult rats. Brain Res 600:169-173.

Gozal E, Row BW, Schurr A, Gozal D (2001) Developmental differences in cortical and hippocampal vulnerability to intermittent hypoxia in the rat. Neurosci Lett 305:197-201.

Greenberg HE, Sica A, Batson D, Scharf SM (1999) Chronic intermittent hypoxia increases sympathetic responsiveness to hypoxia and hypercapnia. J Appl Physiol 86:298-305.

Gutierrez CJ, Harrow J, Haines F (2003) Using an evidence-based protocol to guide rehabilitation and weaning of ventilator-dependent cervical spinal cord injury patients. J Rehabil Res Dev 40:99-110.

Hadley SD, Walker PD, Goshgarian HG (1999) Effects of the serotonin synthesis inhibitor p-CPA on the expression of the crossed phrenic phenomenon $4 \mathrm{~h}$ following C2 spinal cord hemisection. Exp Neurol 160:479-488.

Hains BC, Fullwood SD, Eaton MJ, Hulsebosch CE (2001a) Subdural engraftment of serotonergic neurons following spinal hemisection restores spinal serotonin, downregulates serotonin transporter, and increases BDNF tissue content in rat. Brain Res 913:35-46.

Hains BC, Johnson KM, McAdoo DJ, Eaton MJ, Hulsebosch CE (2001b) Engraftment of serotonergic precursors enhances locomotor function and attenuates chronic central pain behavior following spinal hemisection injury in the rat. Exp Neurol 171:361-378.

Hains BC, Everhart AW, Fullwood SD, Hulsebosch CE (2002) Changes in serotonin, serotonin transporter expression and serotonin denervation supersensitivity: involvement in chronic central pain after spinal hemisection in the rat. Exp Neurol 175:347-362.

Hains BC, Willis WD, Hulsebosch CE (2003) Serotonin receptors 5- $\mathrm{HT}_{1 \mathrm{~A}}$ and $5-\mathrm{HT}_{3}$ reduce hyperexcitability of dorsal horn neurons after chronic spinal cord hemisection injury in rat. Exp Brain Res 149:174-186.

Hashimoto T, Fukuda N (1991) Contribution of serotonin neurons to the functional recovery after spinal cord injury in rats. Brain Res 539:263-270.

Itami C, Kimura F, Kohno T, Matsuoka M, Ichikawa M, Tsumoto T, Nakamura S (2003) Brain-derived neurotrophic factor-dependent unmasking of "silent" synapses in the developing mouse barrel cortex. Proc Natl Acad Sci USA 100:13069-13074.

Kim JH, Udo H, Li HL, Youn TY, Chen M, Kandel ER, Bailey CH (2003) Presynaptic activation of silent synapses and growth of new synapses contribute to intermediate and long-term facilitation in Aplysia. Neuron 40:151-165.

Kinkead R, Mitchell GS (1999) Time-dependent hypoxic ventilatory re- 
sponses in rats: effects of ketanserin and 5-carboxamidotryptamine. Am J Physiol 277:R658-R666.

Ko ML, King MA, Gordon TL, Crisp T (1997) The effects of aging on spinal neurochemistry in the rat. Brain Res Bull 42:95-98.

Ling L, Fuller DD, Bach KB, Kinkead R, Olson Jr EB, Mitchell GS (2001) Chronic intermittent hypoxia elicits serotonin-dependent plasticity in the central neural control of breathing. J Neurosci 21:5381-5388.

McDonald JW, Becker D, Sadowsky CL, Jane JA, Conturo TE, Schultz LM (2002) Late recovery following spinal cord injury. Case report and review of the literature. J Neurosurg Spine 97:252-265.

McGuire M, Zhang Y, White DP, Ling L (2004) Serotonin receptor subtypes required for ventilatory long-term facilitation and its enhancement after chronic intermittent hypoxia in awake rats. Am J Physiol Regul Integr Comp Physiol 286:R334-R341.

Mills CD, Hains BC, Johnson KM, Hulsebosch CE (2001) Strain and model differences in behavioral outcomes after spinal cord injury in rat. J Neurotrauma 18:743-756.

Mitchell GS, Baker TL, Nanda SA, Fuller DD, Zabka AG, Hodgeman BA, Bavis RW, Mack KJ, Olson Jr EB (2001) Invited review: intermittent hypoxia and respiratory plasticity. J Appl Physiol 90:2466-2475.

Moreno DE, Yu XJ, Goshgarian HG (1992) Identification of the axon pathways which mediate functional recovery of a paralyzed hemidiaphragm following spinal cord hemisection in the adult rat. Exp Neurol 116:219-228.

Nantwi KD, El-Bohy AA, Schrimsher GW, Reier PJ, Goshgarian HG (1999) Spontaneous functional recovery in a paralyzed hemidiaphragm following upper cervical spinal cord injury in adult rats. Neurorehab Neural Repair 13:225-234.
Powell FL, Garcia N (2000) Physiological effects of intermittent hypoxia. High Alt Med Biol 1:125-136.

Raineteau O, Schwab ME (2001) Plasticity of motor systems after incomplete spinal cord injury. Nat Rev Neurosci 2:263-273.

Row BW, Kheirandish L, Neville JJ, Gozal D (2002) Impaired spatial learning and hyperactivity in developing rats exposed to intermittent hypoxia. Pediatr Res 52:449-453.

Row BW, Liu R, Xu W, Kheirandish L, Gozal D (2003) Intermittent hypoxia is associated with oxidative stress and spatial learning deficits in the rat. Am J Respir Crit Care Med 167:1548-1553.

Saruhashi Y, Young W, Perkins R (1996) The recovery of 5-HT immunoreactivity in lumbosacral spinal cord and locomotor function after thoracic hemisection. Exp Neurol 139:203-213.

Serebrovskaya TV (2002) Intermittent hypoxia research in the former Soviet Union and the commonwealth of independent states: history and review of the concept and selected applications. High Alt Med Biol 3:205221.

Steinbusch HW (1981) Distribution of serotonin-immunoreactivity in the central nervous system of the rat-cell bodies and terminals. Neuroscience 6:557-618.

Winslow C, Rozovsky J (2003) Effect of spinal cord injury on the respiratory system. Am J Phys Med Rehabil 82:803-814.

Zabka AG, Behan M, Mitchell GS (2001) Selected contribution: Timedependent hypoxic respiratory responses in female rats are influenced by age and by the estrus cycle. J Appl Physiol 91:2831-2838.

Zhuo M (2000) Silent glutamatergic synapses and long-term facilitation in spinal dorsal horn neurons. Prog Brain Res 129:101-113. 